\title{
Modeling the optical constants of hexagonal GaN, InN, and AIN
}

\author{
Aleksandra B. Djurišića) and E. Herbert $\mathrm{Li}^{\mathrm{i})}$ \\ Department of Electrical \& Electronic Engineering, University of Hong Kong, Pokfulam Road, Hong Kong
}

(Received 1 June 1998; accepted for publication 18 November 1998)

\begin{abstract}
Optical constants of hexagonal GaN (in the range 1.5-10 eV), InN (in the range 2-10 eV), and AlN (in the range 6-20 eV) for $E \perp c$ are modeled using a modification of Adachi's model of optical properties of semiconductors. Model parameters are determined using the acceptance-probability-controlled simulated annealing method. The employed model uses an adjustable broadening function instead of the conventional Lorentzian one. The broadening can vary over a range of functions with similar kernels but different wings. Therefore, excessive absorption inherent to Lorentzian broadening due to the large wings of a Lorentz function can be reduced, yielding better agreement with experimental data. As a result, excellent agreement with experimental data is obtained; the relative rms errors for the real part of the index of refraction are below $2 \%$ for all three materials, and, for the imaginary part, below $5 \%$ for $\mathrm{GaN}$ and below $3 \%$ for InN and AlN. (C) 1999 American Institute of Physics. [S0021-8979(99)00605-2]
\end{abstract}

\section{INTRODUCTION}

The nitrides are characterized by their high ionicity, very short bond lengths, low compressibility and high thermal conductivity. III-V nitride semiconductors have attracted much attention because of their potential use in device applications in the visible and near ultraviolet ranges. ${ }^{1}$ Since the fundamental band gaps of $\mathrm{InN}, \mathrm{GaN}$, and $\mathrm{AlN}$ are around $1.9,3.5$, and $6.2 \mathrm{eV}$ respectively, their ternary systems are of interest for electroluminescence devices operating at wavelengths from orange to ultraviolet. ${ }^{2}$ For the application of $\mathrm{GaN}$, InN, and AlN in optoelectronic devices, detailed knowledge of their optical properties is necessary. Experimental studies have mostly been limited to photoluminescence and photoreflectance measurements for the investigation of the position of the fundamental band gap, while work on the determination of the optical constants in the wide spectral range has been scarce. There have been several experimental studies on the optical constants of $\mathrm{GaN}^{3-6}$ and AlN,${ }^{7-9}$ while for InN, data in the wide spectral range have been reported only by Guo et al. ${ }^{10,11}$

It is well known that the optical properties of solids can be described in terms of the complex dielectric function $\epsilon(\omega)=\epsilon_{1}(\omega)+i \epsilon_{2}(\omega)$. In the design of optoelectronic devices, such as lasers and waveguide devices, interplay between the physical dimensions of the device and the index of refraction requires that the index of refraction be known as a function of the wavelength as precisely as possible. The model for the index of refraction should be simple and concise and yet comprehensive enough to account for all the relevant features of the index of refraction over the wide energy range.

Several attempts have been made to model the dielectric function of hexagonal $\mathrm{GaN}$ in narrow spectral range, ${ }^{4,12,13}$ as well as in wide spectral range. ${ }^{3,14,15}$ In the wide spectral

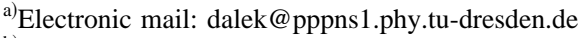

${ }^{b)}$ Electronic mail: ehli@eee.hku.hk
}

range, both the semiempirical tight-binding approach in calculating the dielectric function ${ }^{14,15}$ and Adachi's model dielectric function $(\mathrm{MDF})^{3}$ are available. However, there exist significant discrepancies between the calculated and experimental values of the dielectric function and the index of refraction. Band structure calculations for $\mathrm{InN}^{2,16-18}$ and $\mathrm{AlN}^{17-20}$ have been reported. However, calculations of the optical properties for both $\mathrm{InN}^{17,21}$ and $\mathrm{AlN}^{17,22}$ have been scarce. Foley and Tansley ${ }^{21}$ used the pseudopotential method to calculate the dielectric function of InN, while Christensen and Gorczyca ${ }^{17}$ and Solanki et al..$^{22}$ used the linear-muffintin orbital method. However, optical constants calculated in such a manner agree with experiment only in terms of the position of peaks in the absorption spectrum, while the shape of the curve is completely different (theoretical calculations produce sharper and stronger peaks than those observed in the experimental data). Therefore, the main aim of this work is to model the optical constants of hexagonal $\mathrm{GaN}, \mathrm{InN}$, and AlN for perpendicular polarization $(E \perp c)$ using relatively simple analytical expressions, giving good agreement with experimental data. This should facilitate more efficient and more reliable optoelectronic device design.

The paper is organized as follows. In Sec. II, we describe the employed model of the optical constants of hexagonal semiconductors. In Sec. III, the calculated results are presented and a discussion on the comparison between the experimental data and previous calculations for $\mathrm{GaN}$ is given.

\section{DESCRIPTION OF THE MODEL}

We shall briefly describe the employed model. The complex dielectric function as a function of energy $E=\hbar \omega$ is described by the sum of terms corresponding to one-electron contributions at critical points $E_{0}$ and $E_{1 \beta}$ (where $\beta$ $=A, B, C), \epsilon_{0}(E)$ and $\epsilon_{1}(E)$, excitonic contributions at those critical points, $\epsilon_{0 X}(E)$ and $\epsilon_{1 X}(E)$, and additive constant $\epsilon_{\infty}$ : 


$$
\epsilon(E)=\epsilon_{0}(E)+\epsilon_{0 X}(E)+\epsilon_{1}(E)+\epsilon_{1 X}(E)+\epsilon_{\infty} .
$$

Under the parabolic band assumption, the contribution of three-dimensional $M_{0}$ critical point $E_{0}$ is given by

$$
\epsilon_{0}(E)=A E_{0}^{-3 / 2} \chi_{0}^{-2}\left[2-\left(1+\chi_{0}\right)^{1 / 2}-\left(1-\chi_{0}\right)^{1 / 2}\right],
$$

where

$$
\chi_{0}=\frac{E+i \Gamma_{0}}{E_{0}}
$$

while $A$ and $\Gamma_{0}$ are the strength and damping constants of the $E_{0}$ transition respectively. Normally, one should calculate the separate contributions from $E_{0 \beta}, \beta=A, B, C$ critical points, but due to very small splitting energies among these critical points, $E_{0 \beta}$ can be treated as a single degenerate one. ${ }^{3}$

Exciton contributions at $E_{0}$ critical points are given by

$$
\epsilon_{0 X}(E)=\sum_{m=1}^{\infty} \frac{A_{0}^{e x}}{m^{3}} \frac{1}{E_{0}-\left(G_{0}^{3 \mathrm{D}} / m^{2}\right)-E-i \Gamma_{0}},
$$

where $A_{0}^{e x}$ is the three-dimensional (3D) exciton strength parameter and $G_{0}^{3 \mathrm{D}}$ is the $3 \mathrm{D}$ exciton binding energy.

Contributions of the two-dimensional (2D) $M_{0}$ critical points $E_{1 \beta}$ are given by

$$
\epsilon_{1}(E)=-\sum_{\beta=A, B, C} B_{1 \beta} \chi_{1 \beta}^{-2} \ln \left(1-\chi_{1 \beta}^{2}\right)
$$

where

$$
\chi_{1 \beta}=\frac{E+i \Gamma_{1 \beta}}{E_{1 \beta}}
$$

and $B_{1 \beta}$ and $\Gamma_{1 \beta}$ are the strengths and damping constants of the $E_{1 \beta}$ transitions, respectively.

Contributions of the Wannier type 2D excitons (discrete series of exciton lines at the $E_{1 \beta}$ critical points are given by

$$
\begin{aligned}
\epsilon_{1 X}(E)= & \sum_{\beta=A, B, C,} \sum_{m=1}^{\infty} \frac{B_{1 \beta}^{X}}{(2 m-1)^{3}} \\
& \times \frac{1}{E_{1 \beta}-\left[G_{1 \beta}^{2 \mathrm{D}} /(2 m-1)^{2}\right]-E-i \Gamma_{1 \beta}},
\end{aligned}
$$

where $B_{1 \beta}^{X}$ and $G_{1 \beta}^{2 \mathrm{D}}$ are the strengths and binding energies of the excitons at $E_{1 \beta}$ respectively.

One of the major shortcomings of the above described model is that it is based on the assumption of Lorentzian broadening. The fact that Lorentzian broadening does not accurately describe the absorption processes, especially in the $E_{0}$ critical point region, has already been recognized. ${ }^{23-27}$ Let us address the lifetime broadening problem in greater detail. The dielectric function of a solid, with broadening described by a damping function $\gamma(s)$, is given as follows: ${ }^{23}$

$$
\begin{aligned}
\epsilon(\omega)= & 1+i \frac{2 \hbar^{2} e^{2}}{\epsilon_{0} m^{2}} \sum_{c, v} \int J_{c v}(E) d E\left(\frac{P_{c v}(E)}{E}\right)^{2} \\
& \times\left(\int_{0}^{\infty} d s \exp [i(\hbar \omega-E+i \gamma(s)) s]\right. \\
& \left.-\int_{0}^{\infty} d s \exp \{i[\hbar \omega+E+i \gamma(s)] s\}\right)
\end{aligned}
$$

where subscripts $c$ and $v$ indicate the conduction and valence bands respectively, $J_{c v}(E)$ is the joint density of states, and $P_{c v}(E)$ is the weighted-average matrix element of the momentum operator. If a damping function $\gamma(s)$ is expanded into a power series in $s=t / \hbar$, where $t$ is time, $\gamma(s)=\Gamma$ $+\sigma^{2} s+\cdots$, one usually retains only the first term (Lorentzian broadening) or the second term (Gaussian broadening). In the former case, the broadening function $\Phi$ is given by ${ }^{24}$

$\Phi_{L}=-i \int_{0}^{\infty} d s \exp [i(\hbar \omega \pm E+i \Gamma) s]=\frac{1}{\hbar \omega \pm E+i \Gamma}$.

In the latter case, the broadening function takes the following form:

$$
\begin{aligned}
\Phi_{G} & =-i \int_{0}^{\infty} d s \exp \left[i\left(\hbar \omega \pm E+i \sigma^{2} s\right) s\right] \\
& =-i \frac{\sqrt{\pi}}{2 \sigma} \exp \left[-(\hbar \omega \pm E)^{2} / 4 \sigma^{2}\right]\left[1+\operatorname{erf}\left(i \frac{\hbar \omega \pm E}{2 \sigma}\right)\right] .
\end{aligned}
$$

It has been shown that Gaussian broadening is a much better approximation for the broadening caused by electronphonon and electron-impurities scattering, ${ }^{23,24}$ but in this case, the integration in equation Eq. (8) cannot be performed over the energy domain in an analytically closed form. In order to overcome this problem, Kim et al. ${ }^{23}$ have replaced the damping constant $\Gamma$ in their model with frequency dependent damping constant $\Gamma^{\prime}$ given by

$$
\Gamma^{\prime}(E)=\Gamma \exp \left[-\alpha\left(\frac{E-E_{g}}{\Gamma}\right)^{2}\right],
$$

where $\alpha$ and $\Gamma$ are adjustable model parameters, while $E_{g}$ is the energy of the critical point at which transition occurs. It is shown that this functional representation can closely mimic Gaussian line broadening, while the integration in Eq. (8) can be performed analytically. This is illustrated in Fig. 1 , which depicts the different broadening functions including Lorentzian and Gaussian ones as defined by the imaginary parts of equations (9) and (10) respectively. It can be observed that by varying the $\alpha / \Gamma$ ratio, one can interpolate between the cases of Lorentzian and Gaussian broadening and obtain broadening function with even lower wings than Gaussian. The impact of variable broadening to the dielectric function is illustrated in Fig. 2 for the simple case of a single Lorentzian oscillator. The dielectric function of the oscillator is given by

$$
\epsilon(\omega)=\frac{F_{j}}{\omega^{2}-\omega_{j}^{2}-i \omega \Gamma_{j}},
$$




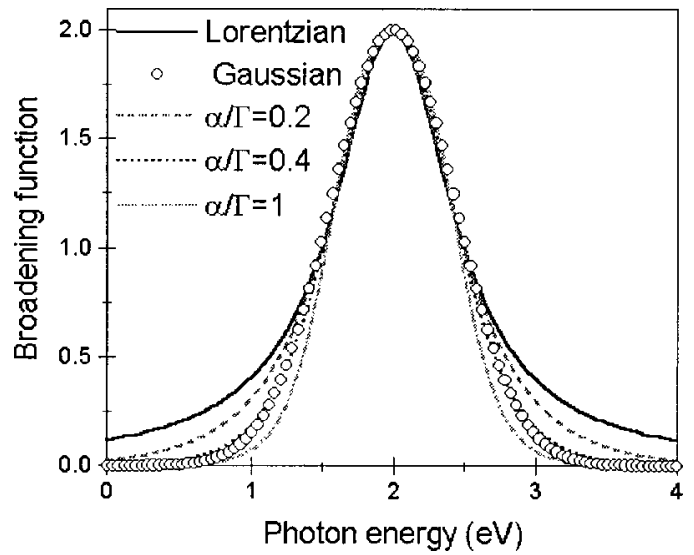

FIG. 1. Illustration of the influence of frequency dependent damping: comparison of different broadening functions.

where $\omega_{j}$ is oscillator frequency, $1 / \Gamma_{j}$ is the lifetime, while $F_{j}=f_{j} \omega_{j}^{2}$ is the parameter associated with oscillator strength $f_{j}$. It can be observed that narrower absorption line corresponds to a larger change in the real part of the dielectric function. The same result can be obtained by a convolution of Lorentzian and Gaussian lines, which can be determined analytically in a closed form ${ }^{25}$ or calculated numerically. ${ }^{26}$ Since it is difficult to determine a priori which broadening mechanism is dominant in an experimentally established absorption line, the frequency dependent damping concept proposed by Kim et al. ${ }^{23}$ represents a simple and yet effective method to model the experimental dielectric function data accurately regardless of the broadening mechanism involved. However, their model is rather complicated, having a large number of parameters, while the equations are material dependent, thus preventing it from being widely accepted and used. On the other hand, Rakic and Majewski ${ }^{24}$ have shown that variable broadening applies equally well to Adachi's model of dielectric function of zinc-blende semiconductors, in particular for GaAs and AlAs.

Therefore, we have incorporated the described modification into MDF for hexagonal semiconductors by replacing the damping constants $\Gamma_{j}(j=0,1 A, 1 B, 1 C)$ in Eqs. (2)-(7) with the expression given by Eq. (11). The model employed

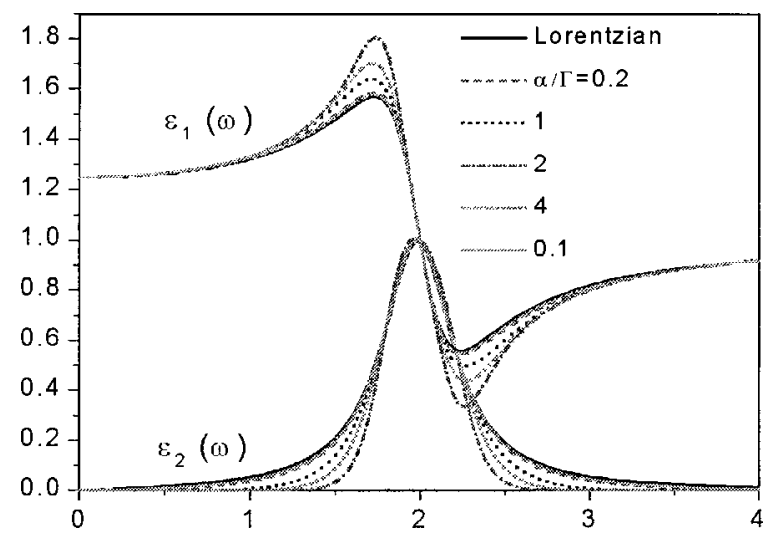

FIG. 2. Illustration of the influence of frequency dependent damping: comparison of the real and imaginary parts of the dielectric function of a single oscillator for different broadening functions. in this work departs from the MDF proposed for the modeling of the optical constants of hexagonal $\mathrm{GaN}$ by Kawashima et $a .^{3}$ on two additional points. First, we take into account not only the ground-state excitons $(m=1)$, but also excited state excitonic contributions $(m>1)$. Second, we include one-electron contributions at $E_{1 \beta}$ critical points given by Eq. (5), which have been disregarded in the calculations of Kawashima et $a l .{ }^{3}$ These three features of our model, adjustable broadening, the summation of excitonic contributions in equations (4) and (7) until the contribution of the next excitonic state becomes less than $10^{-4}$, and considering the oneelectron contributions at $E_{1 \beta}$ critical points, enable us to obtain a significant improvement in the agreement with experimental data over the previously employed MDF.

\section{RESULTS AND DISCUSSION}

The model parameters are obtained by the acceptanceprobability-controlled simulated annealing algorithm, ${ }^{28}$ through minimizing the following objective function:

$$
F=\sum_{i=1}^{N}\left(\left|\frac{n\left(\omega_{i}\right)}{n^{\operatorname{expt}}\left(\omega_{i}\right)}-1\right|+\left|\frac{k\left(\omega_{i}\right)}{k^{\operatorname{expt}}\left(\omega_{i}\right)}-1\right|\right)^{2},
$$

where the summation is performed over the available experimental points, and $n^{\text {expt }}\left(\omega_{i}\right), n\left(\omega_{i}\right), k^{\text {expt }}\left(\omega_{i}\right), k\left(\omega_{i}\right)$ are the experimental and calculated values of the real and imaginary parts of the index of refraction at point $\omega_{i}$ respectively. The choice of the objective function can influence the obtained results, and it is imperative to take into account both real and imaginary parts of the dielectric function or the index of refraction. ${ }^{27}$ We choose to fit the refractive index, which gives lower magnitude of change in the objective function. Since certain parameters of the employed algorithm strongly depend on the magnitude of change in the objective function, such a choice of an objective function provides improved numerical stability for the algorithm. The model parameters for the three nitride materials investigated here are given in Table I.

Figure 3 shows the real and imaginary parts of the dielectric function of hexagonal $\mathrm{GaN}$ as a function of energy. The open circles represent the experimental data, ${ }^{3}$ the solid line is the dielectric function calculated using our modifiedMDF, while the broken line represents the results of Kawashima et $_{\text {al. }}{ }^{3}$ and the dotted line represents our calculations for conventional MDF. Figure 4 shows real and imaginary parts of the index of refraction of GaN vs energy. Excellent agreement between our calculations and experimental data can be observed. As an indication of accuracy with respect to experimental values, we have calculated the relative rms errors, which are, in our case, $1.7 \%$ for $n$ and $4.1 \%$ for $k$, while for the results of Kawashima et al. ${ }^{3}$ the errors equal $7.4 \%$ for $n$ and even $22.8 \%$ for $k$. In our calculations for the conventional MDF, the relative rms error for the real part of the index is found to be equal to $2.9 \%$; for the imaginary part, the relative rms error equals $29 \%$. It can be observed from Figs. 3 and 4 that the modified-MDF is clearly superior to the conventional MDF and that our calculations for MDF have better agreement with the experiment 
TABLE I. Model parameter values.

\begin{tabular}{lcrr}
\hline \hline Parameter & GaN & InN & AlN \\
\hline$\epsilon_{\infty}$ & 0.426 & 1.314 & 1.230 \\
$\left.A(\mathrm{eV})^{1.5}\right)$ & 41.251 & 12.256 & 5.648 \\
$\Gamma_{0}(\mathrm{eV})$ & 0.287 & 0.037 & 0.439 \\
$\alpha_{0}$ & 1.241 & 5.345 & 0.465 \\
$E_{0}(\mathrm{eV})$ & 3.550 & 2.247 & 6.222 \\
$B_{1 A}(\mathrm{eV})$ & 0.778 & 0.361 & 0.236 \\
$B_{1 B}(\mathrm{eV})$ & 0.103 & 1.074 & 0.037 \\
$B_{1 C}(\mathrm{eV})$ & 0.920 & 0.007 & 0.230 \\
$B_{1 A}^{X}(\mathrm{eV})$ & 2.042 & 1.243 & 1.393 \\
$B_{1 B}^{X}(\mathrm{eV})$ & 1.024 & 0.471 & 1.655 \\
$B_{1 C}^{X}(\mathrm{eV})$ & 1.997 & 5.528 & 3.234 \\
$\Gamma_{1 A}(\mathrm{eV})$ & 0.743 & 0.052 & 0.064 \\
$\Gamma_{1 B}(\mathrm{eV})$ & 0.428 & 0.012 & 2.045 \\
$\Gamma_{1 C}(\mathrm{eV})$ & 0.440 & 2.698 & 0.411 \\
$\alpha_{1 A}$ & 0.240 & 5.161 & 0.747 \\
$\alpha_{1 B}$ & 0.011 & 0.574 & 0.687 \\
$\alpha_{1 C}$ & 0.005 & 1.108 & 1.913 \\
$G_{1 A}^{2 D}(\mathrm{eV})$ & 0.0003 & 1.198 & 2.880 \\
$G_{1 B}^{2 D}(\mathrm{eV})$ & 0.356 & 0.521 & 0.980 \\
$G_{1 C}^{2 D}(\mathrm{eV})$ & 1.962 & 4.801 & 5.507 \\
$E_{1 A}(\mathrm{eV})$ & 6.010 & 6.400 & 12.055 \\
$E_{1 B}(\mathrm{eV})$ & 8.182 & 8.230 & 12.900 \\
$E_{1 C}(\mathrm{eV})$ & 8.761 & 7.308 & 0.600 \\
$A_{0}^{e x}(\mathrm{eV})$ & 0.249 & 0.001 & 0.060 \\
$G_{0}^{3 D}(\mathrm{eV})$ & 0.030 & 0.024 & \\
\hline \hline
\end{tabular}

than calculations for the same model reported in another study. ${ }^{3}$ Therefore, disagreement with the experimental data in the study of Kawashima et $a .^{3}$ should be attributed both to the inadequacy of the employed model and an inappropriate choice of the fitting method and/or an inappropriate choice of the objective function in the minimization of discrepancies between the calculated and experimental data.

In order to obtain accurate and reliable model parameter values, global optimization routine must be employed to

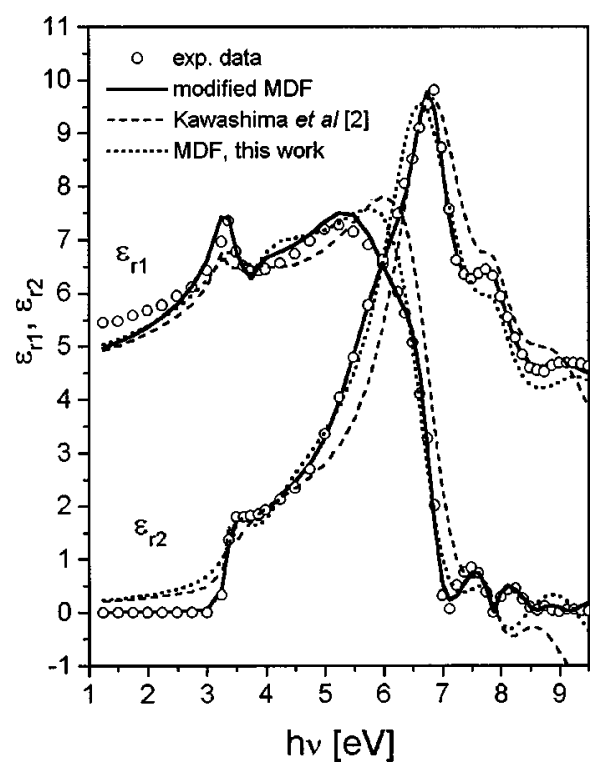

FIG. 3. Real and imaginary part of the dielectric function of GaN vs energy; circles-expt. data, solid line-modified MDF (this work), broken lineMDF (this work), Kawashima et al. (see Ref. 2), dotted line-MDF, this work.

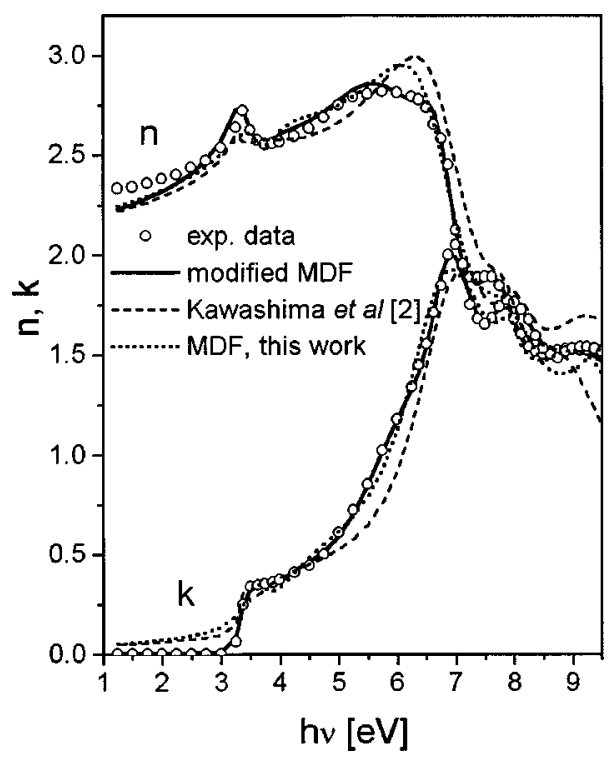

FIG. 4. Real and imaginary part of the index of refraction of GaN vs energy; open circles-expt. data, solid line-modified MDF (this work), broken line-MDF (this work), Kawashima et al. (see Ref. 2), dotted line-MDF, this work.

minimize the discrepancies for both the real and imaginary parts of the dielectric function (or the index of refraction) at the same time. It should be noted that the minimum of such a function does not coincide with the minimum of the sum of mean-square errors for either $n$ or $k$. Also, all these objective functions have a number of local minima, so that conventional downhill methods, like Levenberg-Marqardt or simplex algorithms, often give poor estimates of model parameters. This is because the solution found by conventional downhill methods represents the first minimum encountered in the search from the initial point, i.e., they usually lead to a local minimum close to the given initial values. It is very difficult to provide initial estimates for all the model parameters, except for critical point energies which can be estimated from band structure calculations. Therefore, with conventional optimization methods, it is necessary to rerun the fitting routine many times with different initial parameter values before the acceptable fit is obtained. Even then, there is always a doubt as to whether the obtained solution is indeed the best one. Therefore, to determine accurately the model parameters, one should employ global optimization routine, such as simulated annealing algorithm (which is employed in this work) or genetic algorithms.

Figure 5 shows the real and imaginary parts of the dielectric function of hexagonal $\mathrm{InN}$ as a function of energy. The open circles represent the experimental data of Guo et al., ${ }^{10,11}$ the solid line is the dielectric function calculated using the modified-MDF, while the broken line represents calculations for the MDF. Figure 6 shows the real and imaginary parts of the index of refraction of InN vs energy. Relative rms errors for the real and imaginary parts of the index of refraction are $1.2 \%$ and $2.5 \%$ respectively for the modified-MDF, while for the MDF, they are $1.6 \%$ and $3.1 \%$. It can be observed that in the case of InN, the results obtained by the two models are similar; the modified-MDF 


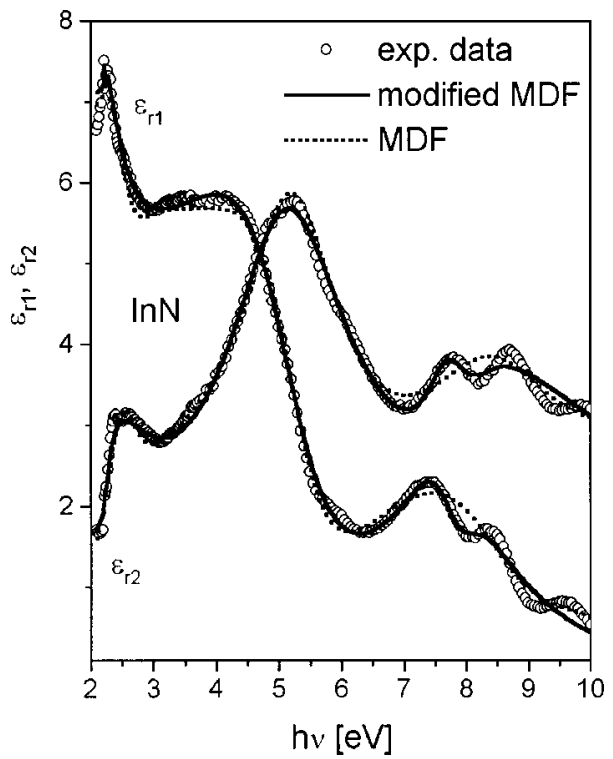

FIG. 5. Real and imaginary part of the dielectric function of InN vs energy; circles-expt. data, solid line-modified MDF, broken line-MDF.

agrees better with the experimental data in the low-energy region, i.e., near the fundamental band gap $E_{0}$, as well as in higher energy regions, i.e., above $6 \mathrm{eV}$.

Figure 7 shows the real and imaginary parts of the dielectric function of hexagonal AlN as a function of energy. The open circles represent experimental data derived from the reflectance data of Guo et al., ${ }^{8}$ the solid line denotes the dielectric function calculated using the modified-MDF, while the broken line represents calculations for the MDF. Figure 8 shows the real and imaginary parts of the index of refraction of AlN vs energy. In this case, relative rms errors for the real and imaginary parts of the index of refraction are $1.5 \%$ and $1.9 \%$, respectively, for the modified-MDF, while for the MDF, they equal $3.1 \%$ and $2.9 \%$. It can be observed that the conventional MDF gives poor agreement with the experi-

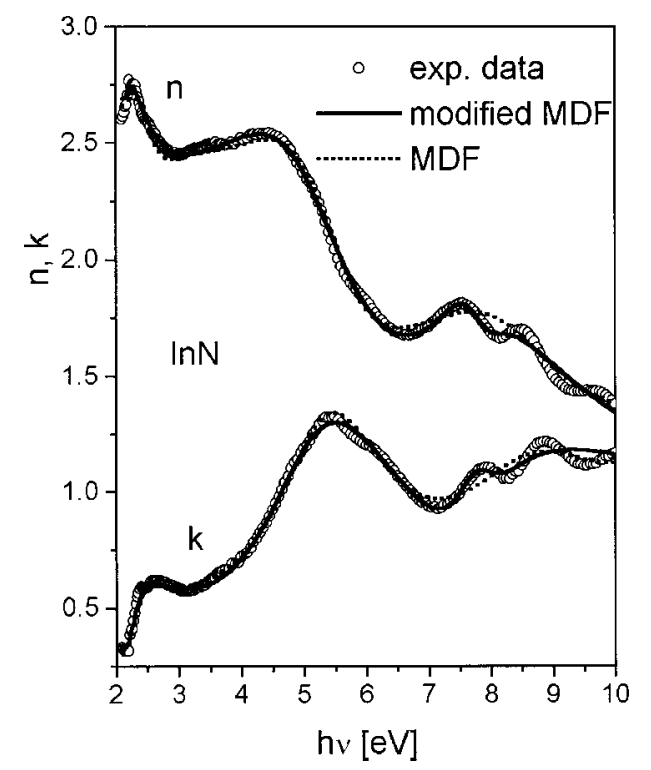

FIG. 6. Real and imaginary part of the index of refraction of InN vs energy; open circles—expt. data, solid line-modified MDF, broken line-MDF.

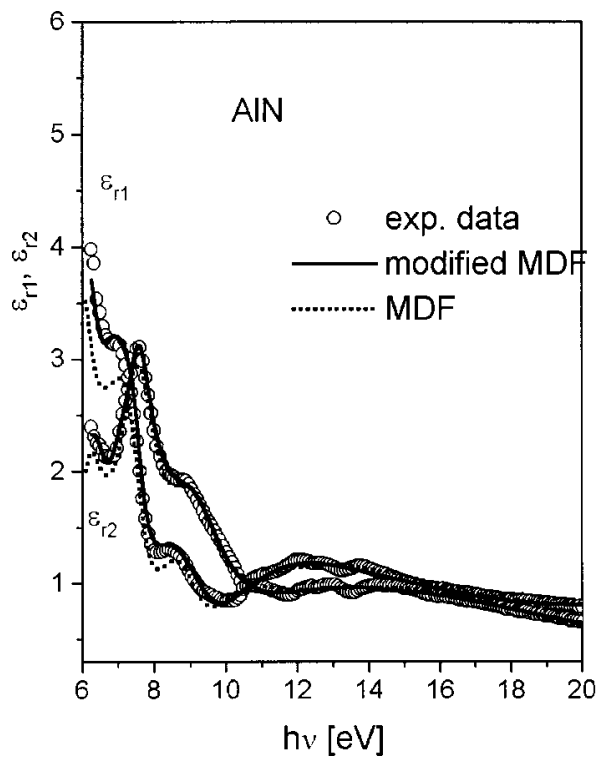

FIG. 7. Real and imaginary part of the dielectric function of AlN vs energy; circles—expt. data, solid line-modified MDF, broken line-MDF.

mental data in the region close to the fundamental band gap $E_{0}$, while the modified-MDF does not have this problem.

The parameters $\Gamma_{0}$ and $\alpha_{0}$ given in Table I for all three nitride materials correspond to a broadening function which is closer to the Gaussian one than to the Lorentzian one, i.e., it does not have large wings characteristic for the Lorentzian broadening function, which result in the excessive absorption in $\epsilon_{2}$. Extended absorption tail caused by Lorentzian broadening assumption in the MDF can be clearly observed in Figs. 3 and 4, while the modified-MDF reproduces accurately the experimental data in the neighborhood and below the fundamental band gap $E_{0}$. For the other two materials, the experimental data are measured above the $E_{0}$, so that the difference between results obtained by the two models is

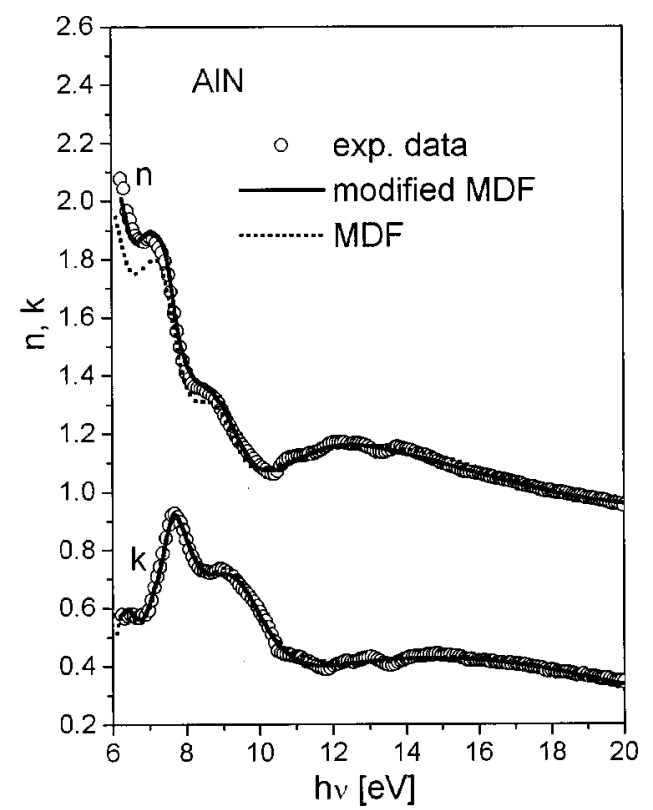

FIG. 8. Real and imaginary part of the index of refraction of AlN vs energy; open circles-expt. data, solid line-modified MDF, broken line-MDF. 
smaller. The results obtained here show that broadening at the fundamental band gap $E_{0}$ should be Gaussian, which is in agreement with Kim et al.'s studies ${ }^{23}$ for GaAs, and Rakić and Majewski's ${ }^{24}$ for GaAs and AlAs.

\section{CONCLUSION}

We have modeled the optical dielectric function of hexagonal $\mathrm{GaN}$ (in the range $1.5-10 \mathrm{eV}$ ), InN (in the range $2-10 \mathrm{eV}$ ) and AIN (in the range $6-20 \mathrm{eV}$ ). The relative rms error obtained for the real part of the index of refraction is below $2 \%$ for all three materials, and for the imaginary part, it is below 5\% for $\mathrm{GaN}$ and below $3 \%$ for InN and AlN. For $\mathrm{GaN}$, we have obtained better agreement with the experimental data than that in a previous study, ${ }^{3}$ in which the relative rms error equals $7.4 \%$ for $n$ and $22.8 \%$ for $k$. For InN and AIN, no previous attempts were made to model the experimental data. There are several reasons for the significant improvement in the accuracy of our calculations. First, our calculations include one-electron contributions at $E_{1 \beta}$ critical points. Second, excited state excitons $(m>1)$ are taken into account. Finally, in the employed model, the type of broadening at each critical point can be varied and it represents an adjustable parameter of the model. From the obtained parameter values for all three materials, we can conclude that the assumption of Lorentzian broadening is not justified at the fundamental band gap $E_{0}$. The excellent agreement with experimental data obtained for all three investigated materials is very encouraging and the next step would be to model their ternary alloy systems.

\section{ACKNOWLEDGMENTS}

This work is supported by the CRCG research grant of the University of Hong Kong. A. B. Djurišić would also like to acknowledge the support of a William Mong Postdoctoral fellowship in the Faculty of Engineering for this work.
${ }^{1}$ S. Nakamura and G. Fasol, The Blue Laser Diode: GaN' Based Light Emitters and Lasers (Springer, Berlin, 1997).

${ }^{2}$ D. W. Jenkins and J. D. Dow, Phys. Rev. B 39, 3317 (1989).

${ }^{3}$ T. Kawashima, H. Yoshikawa, S. Adachi, S. Fuke, and K. Ohtsuka, J. Appl. Phys. 82, 3528 (1997).

${ }^{4}$ S. Logothetidis, J. Petalas, M. Cardona, and T. D. Moustakas, Phys. Rev. B 50, 18017 (1994).

${ }^{5}$ C. G. Olson, D. W. Lynch, and A. Zehe, Phys. Rev. B 24, 4629 (1981).

${ }^{6}$ W. R. Lambrecht, B. Segall, J. Rife, W. R. Hunter, and D. K. Wickenden, Phys. Rev. B 51, 13516 (1995).

${ }^{7}$ H. Yamashita, K. Fukui, S. Misawa, and S. Yoshida, J. Appl. Phys. 50, 896 (1979).

${ }^{8}$ Q. Guo, M. Nishio, H. Ogawa, and A. Yoshida, Phys. Rev. B 55, 15987 (1997).

${ }^{9}$ S. Loughin, R. H. French, W. Y. Ching, Y. N. Xu, and G. A. Slack, Appl. Phys. Lett. 63, 1182 (1993).

${ }^{10}$ Q. Guo, O. Kato, M. Fujisawa, and A. Yoshida, Solid State Commun. 83, 721 (1992).

${ }^{11}$ Q. Guo, H. Ogawa, and A. Yoshida, J. Electron Spectrosc. Relat. Phenom. 79, 9 (1996).

${ }^{12} \mathrm{G}$. Yu, G. Wang, H. Ishikawa, M. Umeno, T. Soga, T. Egawa, J. Watanabe, and T. Jimbo, Appl. Phys. Lett. 70, 3209 (1997).

${ }^{13}$ J. Petalas, S. Logothetidis, S. Boultadakis, M. Alouani, and J. M. Willis, Phys. Rev. B 52, 8082 (1995).

${ }^{14}$ Z. Yang and Z. Xu, J. Phys. B 8, 8303 (1996).

${ }^{15}$ Z. Yang and Z. Xu, Phys. Rev. B 54, 17577 (1996).

${ }^{16}$ M.-H. Tsai, D. W. Jenkins, J. D. Dow, and R. V. Kasowski, Phys. Rev. B 38, 1541 (1988).

${ }^{17}$ N. E. Christensen and I. Gorczyca, Phys. Rev. B 50, 4397 (1994).

${ }^{18}$ A. F. Wright and J. S. Nelson, Proc. SPIE 2399, 498 (1995).

${ }^{19}$ A. Kobayashi, O. F. Sankey, S. M. Volz, and J. D. Dow, Phys. Rev. B 28, 935 (1983).

${ }^{20}$ W. Y. Ching and B. N. Harmon, Phys. Rev. B 34, 5305 (1986)

${ }^{21}$ C. P. Foley and T. L. Transley, Phys. Rev. B 33, 1430 (1986).

${ }^{22}$ A. K. Solanki, A. Kashyap, T. Nantiyal, S. Auluck, and M. A. Khan, Solid State Commun. 94, 1009 (1995).

${ }^{23}$ C. C. Kim, J. W. Garland, H. Abad, and P. M. Raccah, Phys. Rev. B 45, 11749 (1992).

${ }^{24}$ A. D. Rakić and M. L. Majewski, J. Appl. Phys. 80, 5509 (1996).

${ }^{25}$ R. Brandel and D. Bormann, J. Appl. Phys. 71, 1 (1992).

${ }^{26}$ A. Franke, A. Stendal, O. Stenzel, and C. von Borczyskowski, Pure Appl. Opt. 5, 845 (1996).

${ }^{27}$ C. C. Kim and S. Sivananthan, J. Appl. Phys. 78, 4003 (1995).

${ }^{28}$ A. B. Djurišić, A. D. Rakić, and J. M. Elazar, Phys. Rev. E 55, 4797 (1997). 